\title{
Aspects of stress in optimal shaft shoulder fillet
}

\author{
Pedersen, Niels Leergaard
}

Published in:

Journal of Strain Analysis for Engineering Design

Link to article, DOI:

10.1177/0309324718763514

Publication date:

2018

Document Version

Peer reviewed version

Link back to DTU Orbit

Citation (APA):

Pedersen, N. L. (2018). Aspects of stress in optimal shaft shoulder fillet. Journal of Strain Analysis for Engineering Design, 53(5), 285-294. https://doi.org/10.1177/0309324718763514

\section{General rights}

Copyright and moral rights for the publications made accessible in the public portal are retained by the authors and/or other copyright owners and it is a condition of accessing publications that users recognise and abide by the legal requirements associated with these rights.

- Users may download and print one copy of any publication from the public portal for the purpose of private study or research.

- You may not further distribute the material or use it for any profit-making activity or commercial gain

- You may freely distribute the URL identifying the publication in the public portal

If you believe that this document breaches copyright please contact us providing details, and we will remove access to the work immediately and investigate your claim. 


\title{
Aspects of stress in optimal shaft shoulder fillet
}

\author{
Niels Leergaard Pedersen \\ Dept. of Mechanical Engineering, Solid Mechanics \\ Technical University of Denmark \\ Nils Koppels Allé, Building 404, DK-2800 Kgs. Lyngby, Denmark \\ email: nlp@mek.dtu.dk
}

\begin{abstract}
Shafts are among the most common machine elements. The typical shape used to reduce stress concentrations are circular arches due to the simplicity. A shaft is typically loaded by axial, bending and torsional load in different combinations. The stress concentration factors are found in tables and charts. The circular design is not optimal from a strength point of view and the strength can be increased by using shape optimization. It is in the present paper shown how the maximum stress from the combined loads can be minimized, when the shape is parameterized using the simple super ellipse. This makes the resulting optimized designs easily transferable to practical design. The stress evaluation is numerically performed using the finite element method using harmonic elements that facilitates an axisymmetric model although the loading is unsymmetric. The stresses are reduced by up to a factor of $15 \%$.
\end{abstract}

Key words:Machine elements, Shaft fillet, Optimization, Axial load, Bending, Torsion, Harmonic FE.

\section{Introduction}

Machine elements are typically standardized and bought from an external supplier e.g. bearing and bolts. For the different elements to function together it is natural that the design follows specific guidelines specified in different standards so that e.g. a gear from one producer can mate with a gear from another producer. Some machine elements cannot be bought off the shelf this is e.g. the case for shafts. The reason is that there is large variations in the specific use; a shaft has to accommodate to many different design constraints such as placements of bearings, gears, belts but also the assembly process must be taken into account. The different failure modes typically taken into consideration when designing a shaft are; maximum allowable stress, fatigue, deflection and critical speed. The design of a shaft often involves many steps in the design phase.

A shaft is typically loaded by three different load types; axial, bending and torsion separately or more generally in combinations. In most cases the shaft size is controlled by the constraints on the maximum allowable deflection and/or rotation at e.g. the position of bearings or gears. But if care is not taken to limit the stress concentrations these will control the shaft durability. The optimal shaft design for minimum stress concentration is straight forward: it is circular with no changes in the diameter, this design however does not facilitate the attachment of power transmitting elements and bearings. The practical shaft design therefore includes 
abrupt diameter changes that with no rounding will cause stress singularities at the sharp corners. With the use of fillets we don't have infinite stresses but a stress concentration described by the stress concentration factor $K_{t}$ (theoretical stress concentration factor). The theoretic stress concentration factor does not include a possible reduction due to the material notch sensitivity, by including this we have the fatigue stress concentration factor $K_{f}$. The stress found using a conventional finite element (FE) program corresponds to the theoretical stress concentration factor $K_{t}$.

The stress concentration factor is typically found in charts in textbooks see e.g. (Pilkey 1997) and (Norton 2000) and also in more recent papers e.g. (Tipton, Sorem \& Rolovic 1996) and (Rolovic, Tipton \& Sorem 2001). The charts are given for circular fillets and for the three different loading situations separately. Many charts are based on experimental results from photo elasticity. The same results can be found using FE Analysis and the agreement is relatively good. The typical procedure for a combined load case is to find the maximum stress resulting from each loading and from these maximum values calculate a reference stress (e.g. von Mises). This is done although the maximum stress is not found in the same point for the different load cases. Using this procedure we end with a conservative estimate of the maximum stress.

Structural optimization is a mature science and optimization has been performed on shafts, but the result of optimization has had limited impact on the practical shaft design, shafts are still mainly designed using the circular fillet design.

The minimization of maximum stress, i.e., reducing the stress concentration factor and thereby increasing the strength of components can be found in many papers. Many older references specifically in relation to fillet design can be found in (Pilkey 1997) more recent references includes (Bhavikatti \& Ramakrishnan 1979), (Pedersen \& Laursen 1982),(Pedersen 2000), (Parvizian \& Fenner 2002), (Van Miegroet \& Duysinx 2007), (Pedersen 2008) and (Sonmez 2009) and references within these papers. The majority of papers deal with flat or circular bars in tension. Despite the large number of publications very little optimization has been reported for the combined loading case. In (Sonmez 2009) an example of combined torsion and transverse load is presented.

In the present paper we will apply similar methods as presented in (Pedersen \& Pedersen 2008), (Pedersen 2011), (Pedersen 2013) and (Pedersen 2015) where optimization is performed on other machine elements such as gears and bolts. Typically relatively large strength improvements can be found for simple design modifications. Central for these design modifications is that revised design can still comply with the design specified by the standards.

The stress concentration evaluation is performed using the FE method. For a successful application of shape optimization a couple of points are important. The main point is that the shape (with the high stress) parameterization is done separately from the design domain FE meshing, i.e. the nodal position in the FE mesh should not be used as design variables, see e.g. (Ding 1986). The shape can be given either analytically or numerically. Which methods that is selected is not important for a successful optimization, the analytically given shape is more easily reported to other researchers whereas a numerical spline based design can be utilized directly on the manufacturing proces through the CAD system. With the nodal position given by these types of parameterization a mesh refinement can be performed to verify that the stress is converged.

In the present paper we will, like in (Tipton et al. 1996) use the ANSYS program (ANSYS 1970 -) and utilize the harmonic elements. This allows for 2D axisymmetric models that can handle the non-axisymmetric loads or out of plane loading associated with bending and torsion. The harmonic elements can also handle the in-plane loading associated with axial load so the 
same model can be used for all three load cases.

The paper is organized as follows. In Section 2 the FE modelling is presented and a discussion of using an axisymmetric model using harmonic elements relative to using a full 3D FE model is discussed. For verification the results are compared with the standard design and the results presented in stress concentration charts, also with the results presented in (Tipton et al. 1996). In Section 3 the suggested parameterization and proposed fillet design modifications are presented. The conclusions are given in Section 4.

\section{FE modelling of shaft fillet}

Fatigue strength evaluation of components (machine elements) makes the modelling and estimation of stress concentrations the important point. This evaluation demands a high number of FE meshing nodes on the boundary shape where the stress concentration is present. For a given design e.g. the standard circular fillet shape the position of high stress can be found and the element refinement can be done locally at this specific point reducing the need for overall mesh refinement considerably. In optimization where we seek to lower the maximum stress the point of maximum stress is not known but is a function of the design. In fact the optimality criterion for minimum stress on the surface/shape is that the stress is constant on the surface, which will increase the number of nodes on the boundary since we need to evaluate the stress (correctly) over a larger area.

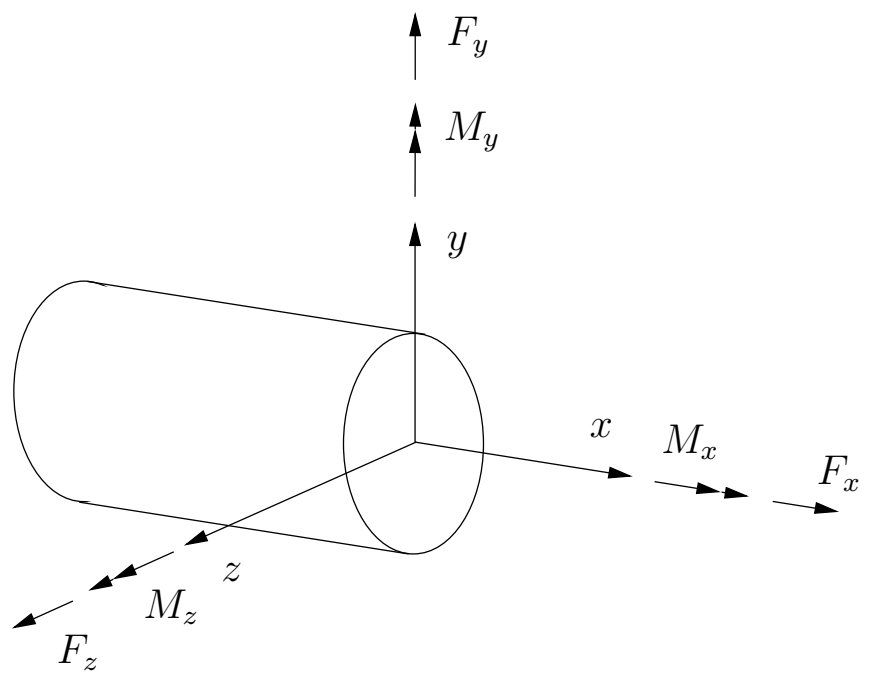

Figure 1: Definitions of forces and moments on a beam cross section.

In Figure 1 the possible forces and moments on a shaft are shown, axial load $F_{x}$, torsional load $M_{x}$. The bending moment is $M_{b}=\sqrt{M_{y}^{2}+M_{z}^{2}}$. The total shear force $F_{s}=\sqrt{F_{y}^{2}+F_{z}^{2}}$ is normally not accounted for because the shear stress associated with this force is zero at the point of maximum normal stress due to the bending moment. The shaft geometry is axisymmetric and only the axial load is also axisymmetric.

FE modelling of the shaft can be done in many ways. For the axial load the simplest modelling is to use a standard axisymmetric model. For torsional load the loading is out-of-plane relative to the plane of a 2D axisymmetric model, and this cannot be done with standard axisymmetric modelling. A full 3D shaft modelling can of course be used but due to the symmetry we can reduce the $3 \mathrm{D}$ model to a sector of a circle where the central angle in principle can go to zero but this will be limited by the resulting FE mesh quality. For the bending moment we can 
also utilize symmetry in a 3D FE model, here it is possible to model only one quarter of the shaft (Sector of a circle with central angle $90^{\circ}$ ).

\subsection{Example of maximum shear stress}

The problem with modelling in 3D is best illustrated by a small example. The shaft loading and geometric quantities are as shown in Figure 2.

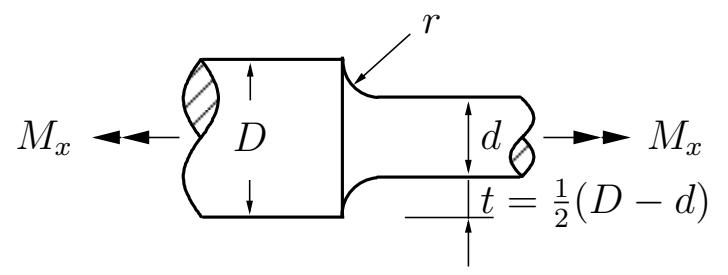

Figure 2: Definition of geometric quantities for a shaft in torsion.

The stress of interest here is the shear stress and the stress concentration factor is defined as

$$
K_{t s}=\frac{\tau_{\max }}{\tau_{\text {nom }}}
$$

where $\tau_{\max }$ is the maximum shear stress and the nominal stress is defined as

$$
\tau_{n o m}=\frac{16 M_{x}}{\pi d^{3}}
$$

A discussion of the definition of maximum shear stress in equation (1) is needed. The maximum stress could be defined as the maximum shear stress value on a cross section as defined by

$$
\sqrt{\tau_{x y}^{2}+\tau_{x z}^{2}}
$$

or the maximum shear stress could be defined as the maximum shear stress defined by the principal stresses $\sigma_{1}, \sigma_{2}$ and $\sigma_{3}$ as

$$
\max \left(\left|\frac{\sigma_{1}-\sigma_{2}}{2}\right|,\left|\frac{\sigma_{2}-\sigma_{3}}{2}\right|,\left|\frac{\sigma_{3}-\sigma_{1}}{2}\right|\right)
$$

In (Rolovic et al. 2001) the later definition is used, and in (Pilkey 1997) it is simply stated that $\tau_{\max }$ is the maximum shear stress indicating that the same definition is used. For comparison we will here use the same definition. It should however be noted that the result of using equation (3) or equation (4) does not yield the same result, and by using equation (4) the maximum shear stress is no longer perpendicular to the axial direction. For shaft in torsion we know that (with reference to the coordinate system defined in Figure 1).

$$
\sigma_{x}=0, \quad \sigma_{y}=0, \quad \sigma_{z}=0, \quad \tau_{r}=0
$$

where $\tau_{r}$ is the radial shear stress. This corresponds to the principal stresses 


$$
\sigma_{1}=-\sigma_{3}, \quad \sigma_{2}=0
$$

from which we find

$$
\tau_{\max }=\sigma_{1 \max }
$$

In the example the following geometry data is used

$$
d=10 \mathrm{~mm}, \quad \quad \quad \quad \quad r=18 \mathrm{~mm}, \quad 1 \mathrm{~mm}
$$

From (Pilkey 1997) we have the following estimate for the stress concentration.

$$
K_{t s}=C_{1}+C_{2}\left(\frac{2 t}{D}\right)+C_{3}\left(\frac{2 t}{D}\right)^{2}+C_{4}\left(\frac{2 t}{D}\right)^{3}
$$

with

$$
\begin{aligned}
& C_{1}=0.905+0.783 \sqrt{t / r}-0.075 t / r \\
& C_{2}=-0.437-1.969 \sqrt{t / r}+0.553 t / r \\
& C_{3}=1.557+1.073 \sqrt{t / r}-0.578 t / r \\
& C_{4}=-1.061+0.171 \sqrt{t / r}+0.086 t / r
\end{aligned}
$$

In (Norton 2000) the following estimate for the stress concentration is given as (values are found from linear extrapolation),

$$
K_{t s}=0.85903\left(\frac{r}{d}\right)^{-0.23655}
$$

With the given data this corresponds to

$$
K_{t s}^{\text {Pilkey }}=1.452, \quad K_{t s}^{\text {Norton }}=1.481
$$

The shaft is modelled in COMSOL with a quarter shaft model. The mesh needs to be refined at the fillet as seen in Figure 3 in order for the stress calculations to be converged. The number of degrees of freedom for the shown model is $5 \cdot 10^{5}$ using tetrahedrals with quadratic displacement assumption. A mesh refinement must be performed to verify the stress level. The number of elements in the FE model can of course be reduced by a fine tuning, but the overall number of degrees of freedom in the 3D FE model will stay around the same number. With the objective of using shape optimization to reduce the maximum stress the number of elements should most probably be increased leading to a slow optimization process. The FE results are shown in Figure 4.

The maximum value corresponds to a stress concentration factor of $K_{t s}=1.39$, i.e. slightly less than what is found from the two references ( $4 \%$ and $6 \%$ less respectively). The result reported in (Rolovic et al. 2001) (based on the overall same approach) is $K_{t s}=1.41$ i.e. in closer 
agreement. If the stress concentration is based on equation (3) the result is $K_{t s}=1.36$, clearly showing the difference between the two definitions of maximum shear stress. The maximum shear stress along the fillet is seen in Figure 5. It is clearly seen that the stress is not optimal due to the large variation and because there is not a segment with constant stress.

Using the harmonic axisymmetric elements in ANSYS for the same design we find the results shown in Figures 5 and 6 . The stress found in the fillet is identically to that found in the $3 \mathrm{D}$ model as seen in Figure 5 so also here the stress concentration factor found is $K_{t s}=1.39$.

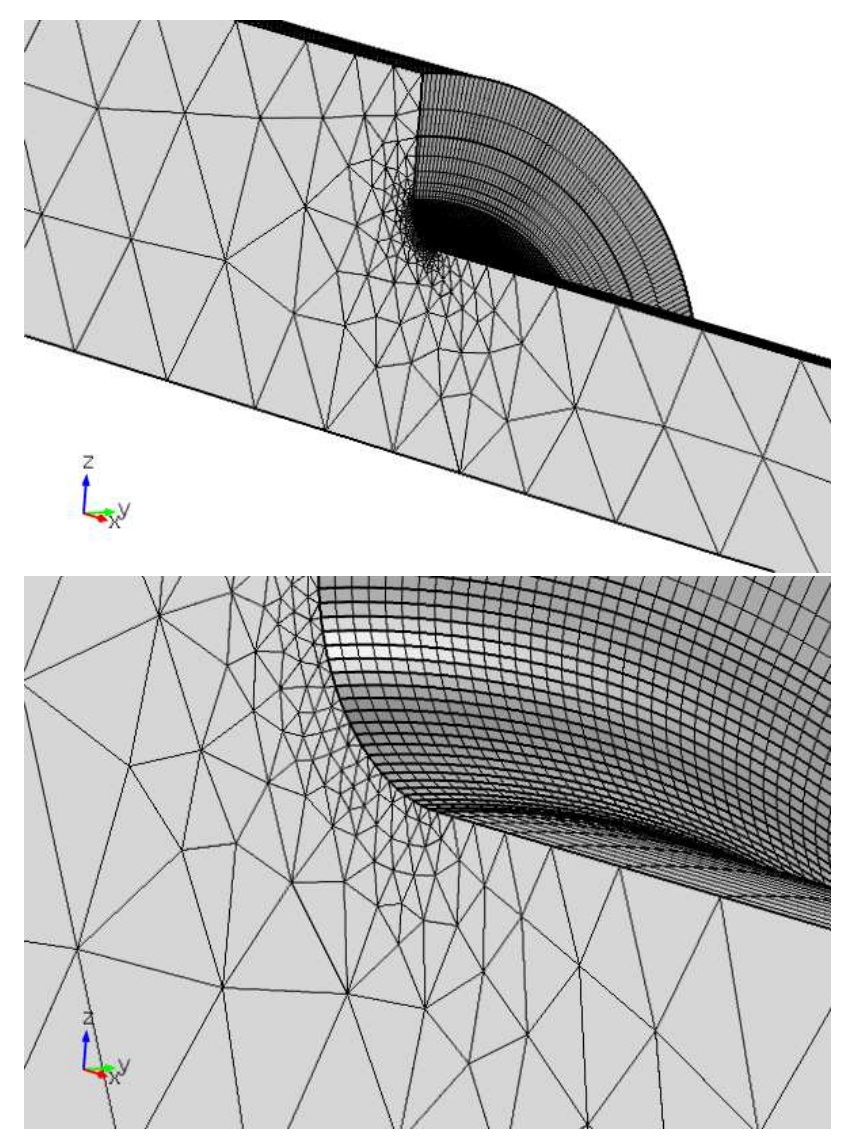

Figure 3: Zoom of FE mesh of shaft.

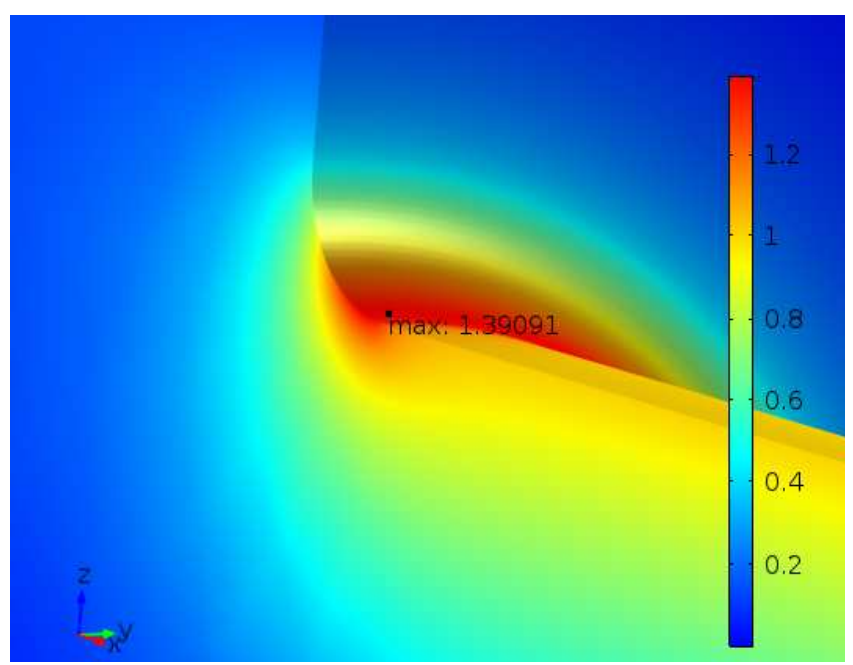

Figure 4: Shear stress in shaft. 


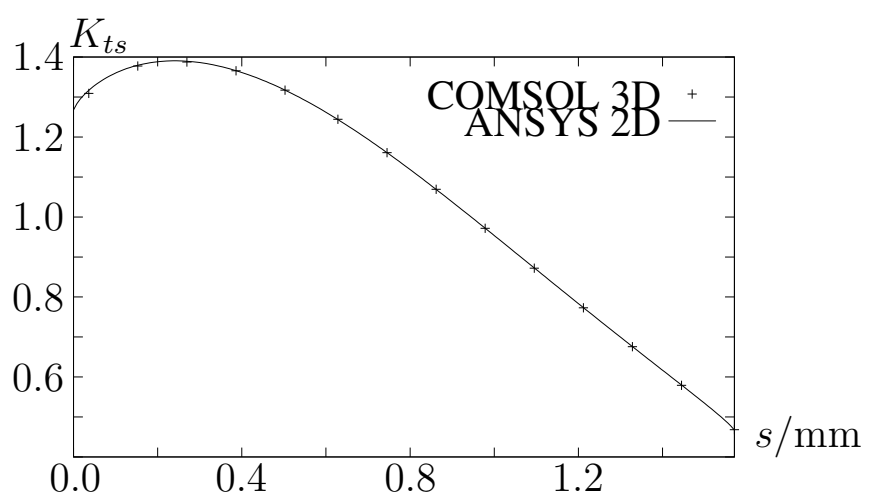

Figure 5: Shear stress concentration factor along fillet in shaft (corresponding to the largest principal stress). Here $s$ is the arc length along the fillet.
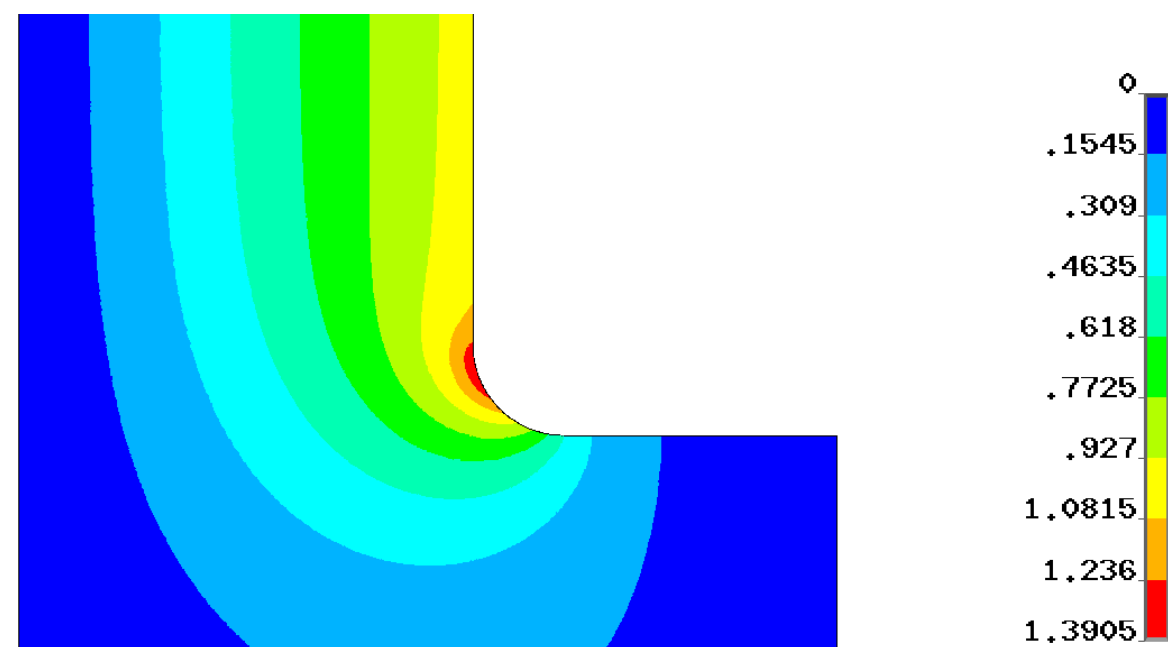

Figure 6: Shear stress in shaft scaled by $\tau_{n o m}$, symmetry line is the left boundary (ANSYS model). 


\subsection{Influence from Poisson's ratio}

In the charts for stress concentration found in the literature it is never, to the authors knowledge, specified what value of Poisson's ratio, $\nu$, that have been used/applied. Many original charts have been made from photoelastic experiments i.e. not using steel, in (Dally \& Riley 1991) it is stated that the influence from Poisson ratio on the result is usually small, meaning less than $7 \%$. In relation to optimization this difference is not negligible since the improvement in the stress level is of the same order typically.

To exemplify the influence from Poisson's ratio we show in Figure 7 the stress concentration for bending defined as

$$
K_{t}=\frac{\sigma_{\max }}{\sigma_{\text {nom }}}
$$

where $\sigma_{\max }$ is the maximum principal stress and the nominal stress is defined as

$$
\sigma_{n o m}=\frac{32 M_{b}}{\pi d^{3}}
$$

for the specific value $D / d=3.0$.

From Figure 7 it is seen that overall the influence from $\nu$ is small but not negligible especially for the low values of $r / d$. In the graph the highest deviation found is

$$
\frac{K_{t}(\nu=0.499)-K_{t}(\nu=0.0)}{K_{t}(\nu=0.3)} \approx 0.1
$$

i.e. a $10 \%$ difference. For the highest value of $r / d$ shown the deviation is approximately $2 \%$. In the previous part of the paper when not specified and in the remaining part of the paper we will assume that $\nu=0.3$ corresponding to the normal value for a steel shaft.

\subsection{Stress concentration charts using harmonic FE}

Using harmonic axisymmetric finite elements is the best numerical method for evaluating the stress concentrations in shaft fillets. This is due to the relatively high number of elements needed along the fillet with the stress concentration. From previous studies of stress concentration, e.g. in gear (Pedersen 2015), it has been shown that up to 400 nodes along the shape are needed in order for the stress level to be converged. It is specifically essential when using FE analysis to evaluate stress concentration that a mesh refinement is performed to verify that the stress level is correct. An automated mesh refinement will typically not make the stress converge because the extra nodes on the boundary are placed on the linear segments between the existing nodes. The new nodes must be placed correctly on the defined shape of the fillet.

The results presented here is compared to what is reported in (Pilkey 1997) from older references and the more reason found in (Tipton et al. 1996) and (Rolovic et al. 2001). In principle the same computational approach as used in (Tipton et al. 1996) and (Rolovic et al. 2001) is used here. The overall findings are however not the same which might be due to the FE mesh used in (Tipton et al. 1996) which compared to the refined mesh used in the present paper is too coarse. In the present paper the number of 8 node elements along the fillet is increased to 400 to verify that the stress level is converged. 
For all three types of loading (axial, bending and torsion) a curve fit to the data have been made using the formulas

$$
K_{t}=A+B\left(\frac{r}{d}\right)^{-C}, \quad K_{t s}=A+B\left(\frac{r}{d}\right)^{-C}
$$

where the curve fitted values of $A, B$ and $C$ are given in tables for different values of $D / d$. In order to have the tight fit between the numerical results and the proposed curve fits it is not attempted to find function that specify the values $A, B$ and $C$ as a function of $D / d$ as done in (Tipton et al. 1996) and (Rolovic et al. 2001).

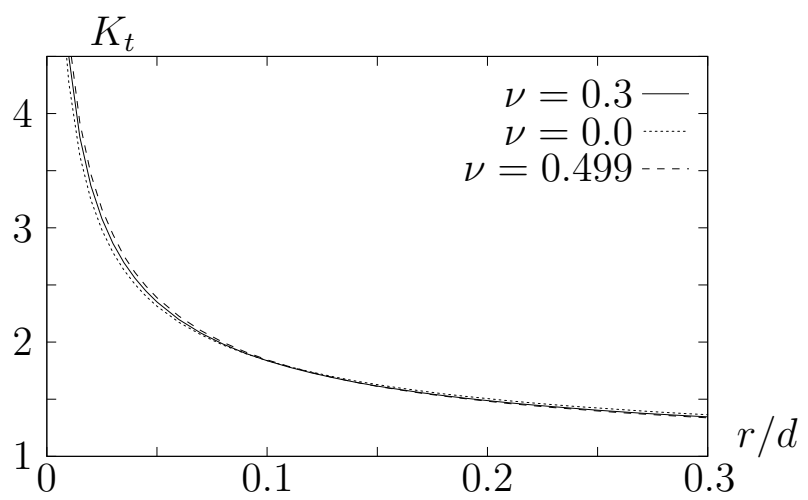

Figure 7: Influence on Poisson's ratio on the stress concentrations for shaft fillet in bending.

\section{Shaft in bending}

In Figure 8 the stress concentration factor for the shaft with a fillet in bending is shown. The points in the figure are the numerical solutions and the full lines are the curve fits. The curve fit is split in two in order to have a tight fit; one fit for $r / d<0.05$ and one for $r / d>0.05$. The values for the individual curve fit that follows equation (15) are given in Table 1. The curve fits are seen to be in excellent agreement with the numerical results for the full range of $r / d$

\begin{tabular}{|c|c|c|c|c|c|c|}
\hline & \multicolumn{3}{|c|}{$r / d<0.05$} & \multicolumn{3}{c|}{$r / d>0.05$} \\
$D / d$ & $A$ & $B$ & $C$ & $A$ & $B$ & $C$ \\
\hline \hline 1.01 & 0.98561 & 0.080796 & 0.50 & 0.98211 & 0.081126 & 0.50 \\
1.02 & 0.95197 & 0.12017 & 0.49 & 0.96578 & 0.11340 & 0.50 \\
1.05 & 0.87457 & 0.19441 & 0.48 & 0.92468 & 0.17214 & 0.50 \\
1.1 & 0.80813 & 0.25766 & 0.48 & 0.87029 & 0.22848 & 0.50 \\
1.2 & 0.71456 & 0.32678 & 0.48 & 0.85527 & 0.24540 & 0.54 \\
1.5 & 0.58723 & 0.40133 & 0.48 & 0.83198 & 0.25526 & 0.58 \\
2.0 & 0.53076 & 0.42869 & 0.48 & 0.82931 & 0.25091 & 0.60 \\
3.0 & 0.51098 & 0.43701 & 0.48 & 0.84857 & 0.23605 & 0.62 \\
\hline
\end{tabular}

Table 1: Values used for curve fits for bending load.

The results here compare very well to that found in (Tipton et al. 1996) which are to be expected because the same overall analysis method is used. In the present paper the load is applied as a triangular stress at the end, not as a load couple as done in (Tipton et al. 1996). Small differences might be due to the meshing quality. In the present paper up to 400 elements have been applied along the fillet to guaranty stress convergence. 


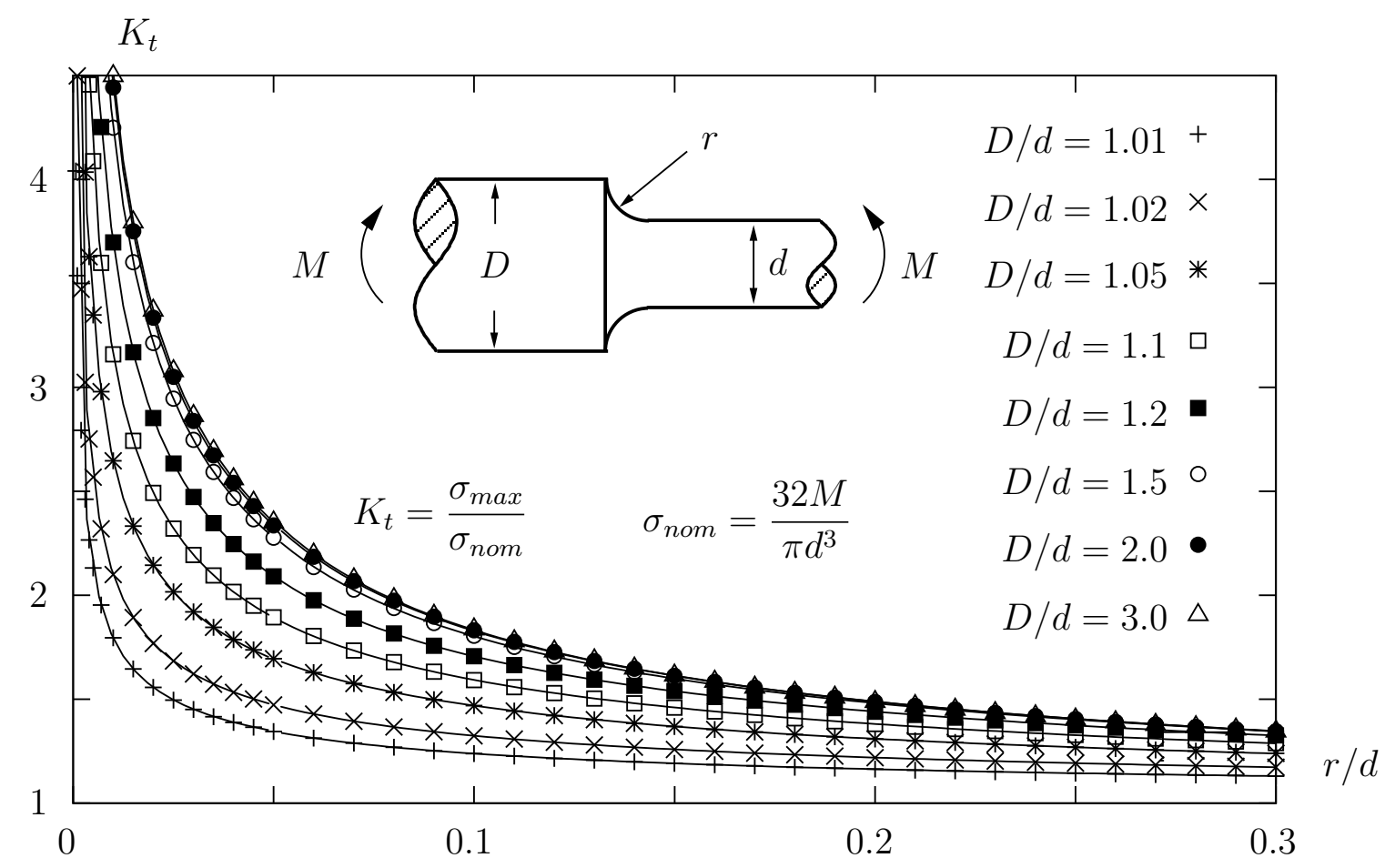

Figure 8: Chart of stress concentrations for shaft fillet in bending, full lines are curve fits and the points are the numerical solutions.

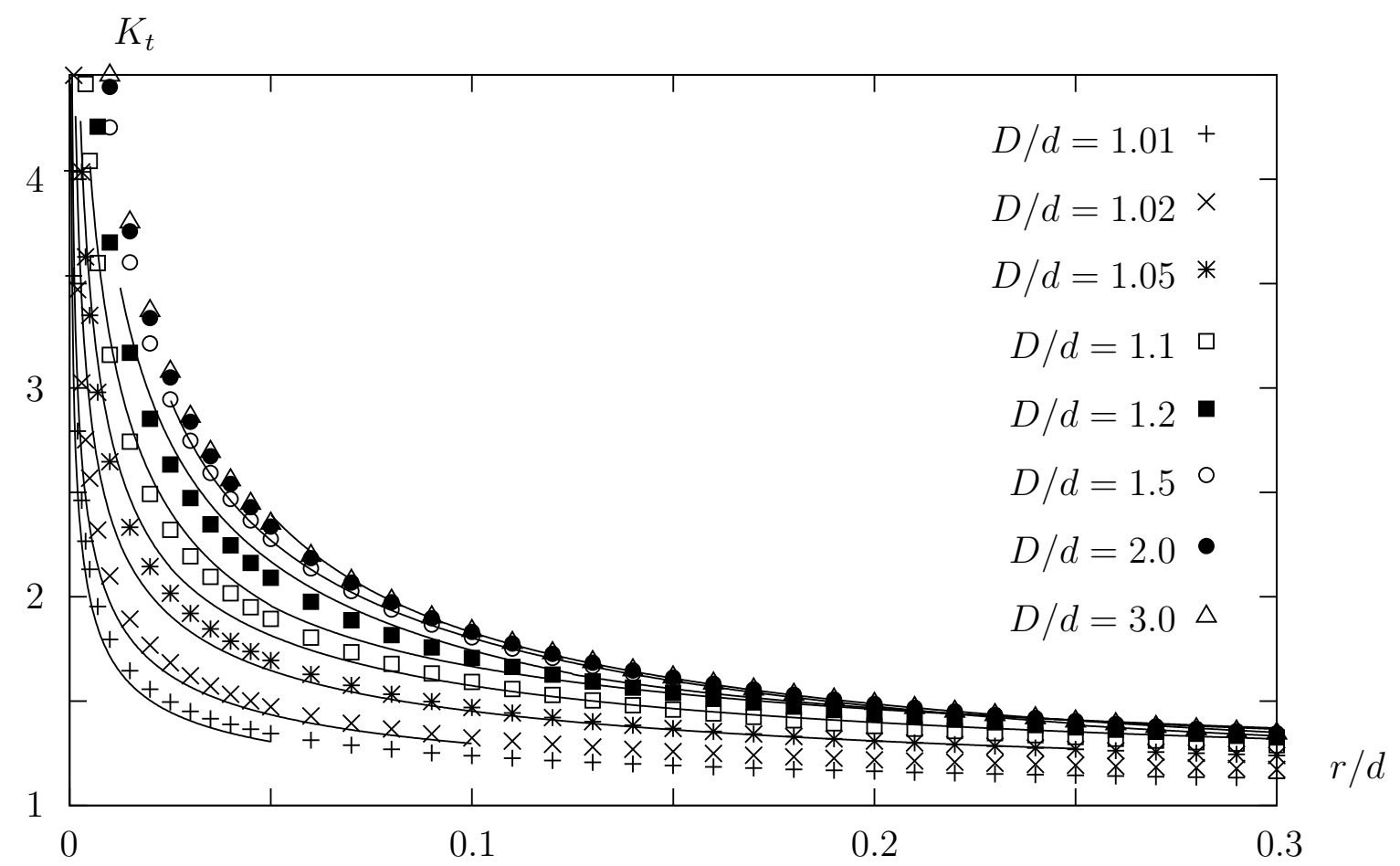

Figure 9: Chart of stress concentrations for shaft fillet in bending, full lines are curve fits from (Pilkey 1997) the points are the present papers numerical results. 
In Figure 9 the curve fits presented in (Pilkey 1997) is compared with the present findings. Overall the results compares well, it is however seen that the curve fit from (Pilkey 1997) generally underestimates the stress concentration factor which potentially can lead to fatigue failure of a design.

\section{Shaft in axial load}

In Figure 10 the stress concentration factor for the shaft with a fillet in axial load is shown. The points in the figure are the numerical solutions and the full lines are the curve fits. The curve fit is split in two in order to have a tight fit; one fit for $r / d<0.05$ and one for $r / d>0.05$. The values for the individual curve fit that follows equation (15) are given in Table 2. The curve fits are seen to be in excellent agreement with the numerical results for the full range of $r / d$. It is noted that compared to bending, axial load generally gives rise to a higher stress concentration factor.

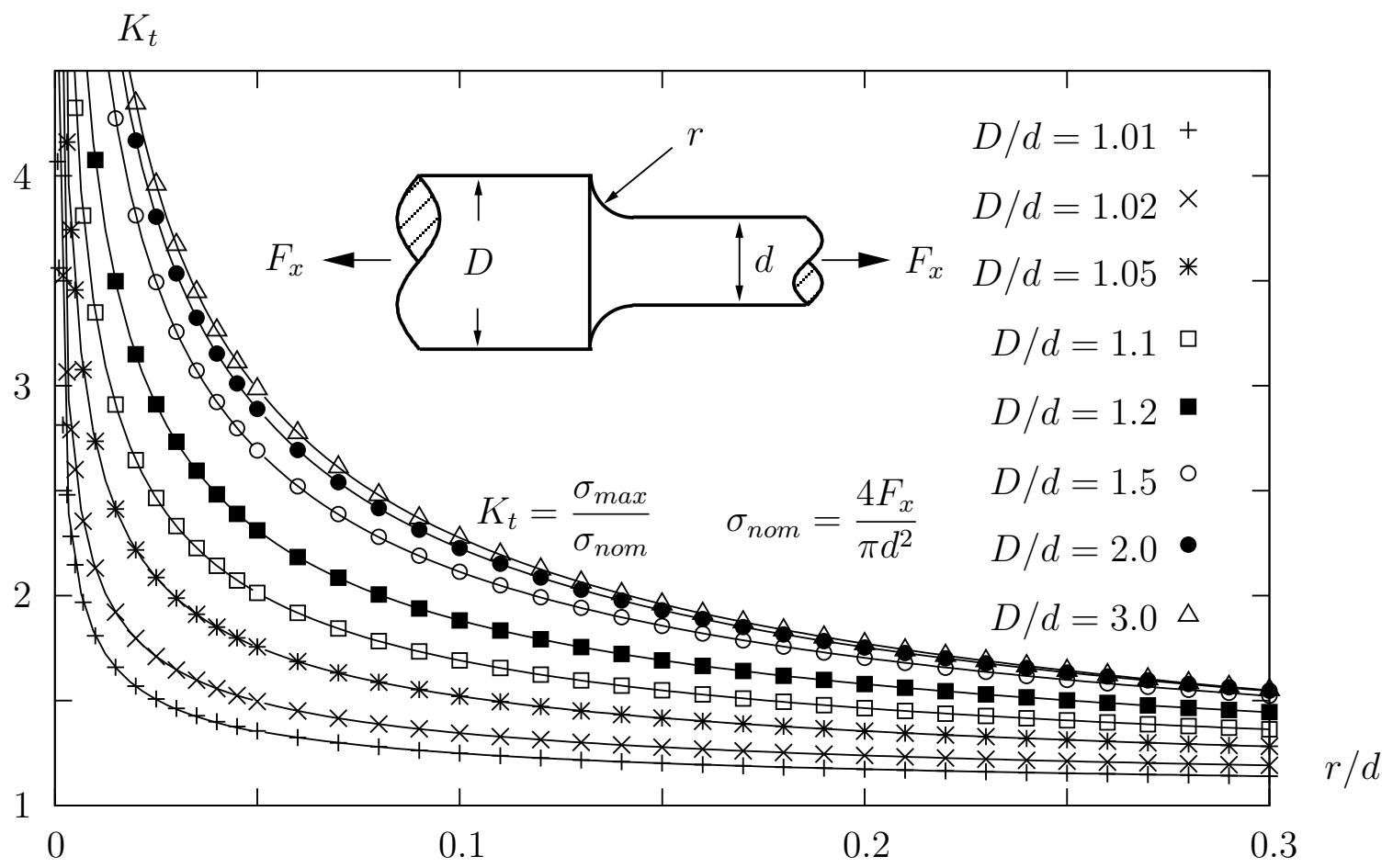

Figure 10: Chart of stress concentrations for shaft fillet in axial load, full lines are curve fits and the points are the numerical solutions.

\begin{tabular}{|c|c|c|c|c|c|c|}
\hline \multirow[b]{2}{*}{$D / d$} & \multicolumn{3}{|c|}{$r / d<0.05$} & \multicolumn{3}{|c|}{$r / d>0.05$} \\
\hline & $A$ & $B$ & $C$ & $A$ & $B$ & $C$ \\
\hline 1.010 .96805 & 0.088152 & 0.49 & 0.99065 & 0.081683 & 0.50 & \\
\hline 1.020 .96858 & 0.12168 & 0.49 & 0.98126 & 0.11508 & 0.50 & \\
\hline 1.050 .91272 & 0.19983 & 0.48 & 0.95415 & 0.17929 & 0.50 & \\
\hline 1.10 .86995 & 0.27156 & 0.48 & 0.91445 & 0.24573 & 0.50 & \\
\hline 1.20 .80018 & 0.35908 & 0.48 & 0.84618 & 0.32766 & 0.50 & \\
\hline 1.50 .61505 & 0.50826 & 0.47 & 0.71395 & 0.44264 & 0.50 & \\
\hline 2.00 .51699 & 0.58066 & 0.47 & 0.61661 & 0.50886 & 0.50 & \\
\hline 3.00 .45837 & 0.61826 & 0.47 & 0.55541 & 0.54394 & 0.50 & \\
\hline
\end{tabular}

Table 2: Values used for curve fits for axial load. 
The results here do not compare so well to the results taken from (Tipton et al. 1996). The comparison is given in Figure 11. It is noted that it is primarily for the low values of $D / d$ that there is a difference, i.e. for $D / d<1.1$. This is most probably due to the different meshes. The results of (Tipton et al. 1996) indicate that a too coarse mesh has been used since the stress is underestimated. The comparison to the curve fit present in (Pilkey 1997) is worse and generally the stress concentrations are underestimated.

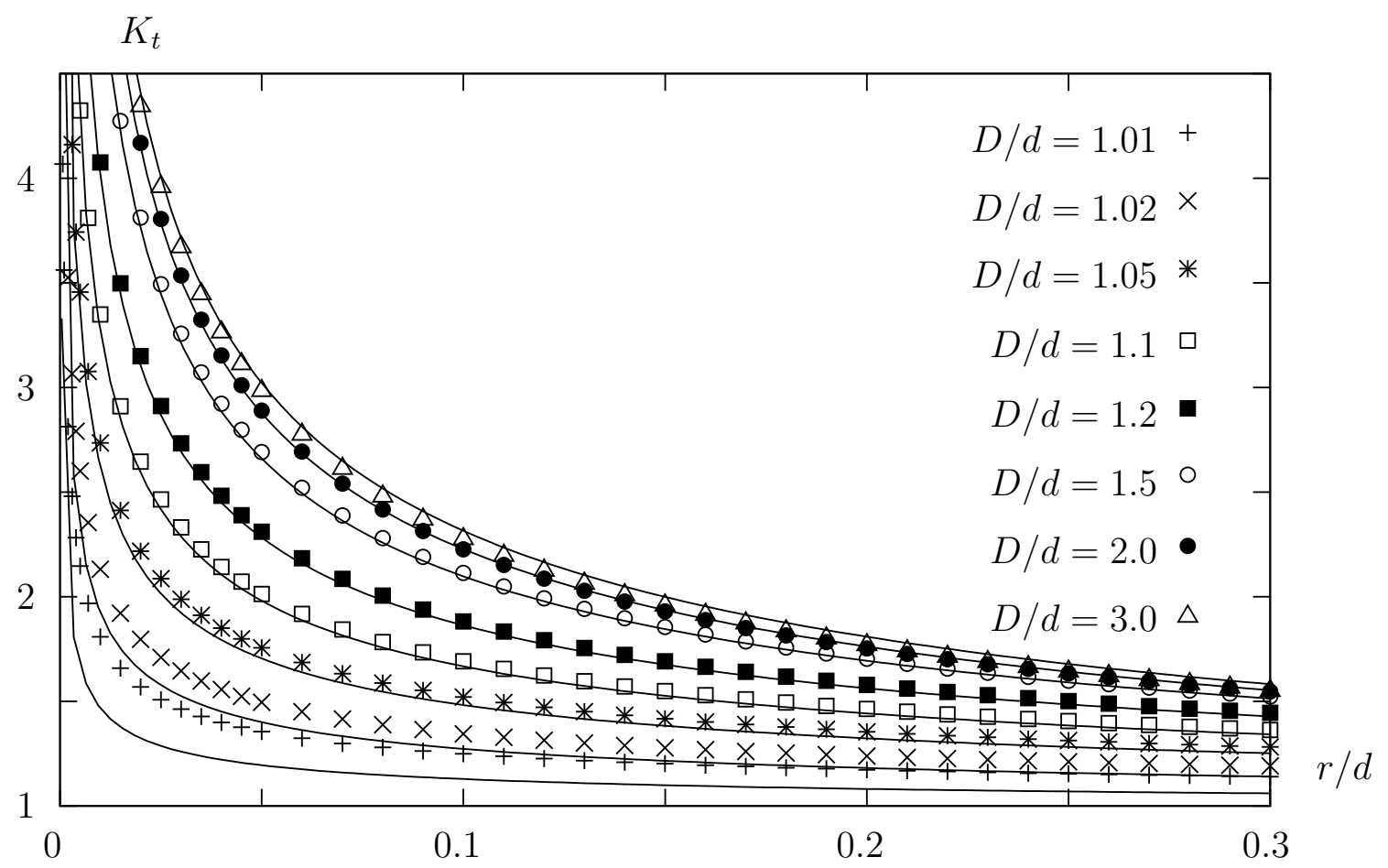

Figure 11: Chart of stress concentrations for shaft fillet in axial load, full lines are curve fits from (Tipton et al. 1996) the points are the numerical solutions from the present paper.

\section{Shaft in torsion}

In Figure 12 the stress concentration factor for the shaft with a fillet in torsion is shown. The points in the figure are the numerical solutions and the full lines are the curve fits. The curve fit is split in two in order to have a tight fit; one fit for $r / d<0.05$ and one for $r / d>0.05$. The values for the individual curve fits that follows equation (15) are given in Table 3 . The curve fits are seen to be in excellent agreement with the numerical results for the full range of $r / d$. It is noted that compared to bending and axial load, torsion generally gives rise to a lower stress concentration factor. It is again noted that the $\tau_{\max }$ is defined from the principal stress as shown in equation (4).

The results here compare very well to that found in (Rolovic et al. 2001) for the full range shown in the figure.

\section{Super elliptical fillet design}

In previous publications see (Pedersen \& Pedersen 2008), (Pedersen 2011), (Pedersen 2013) and (Pedersen 2015) related to stress minimization/optimization in machine elements it has been 


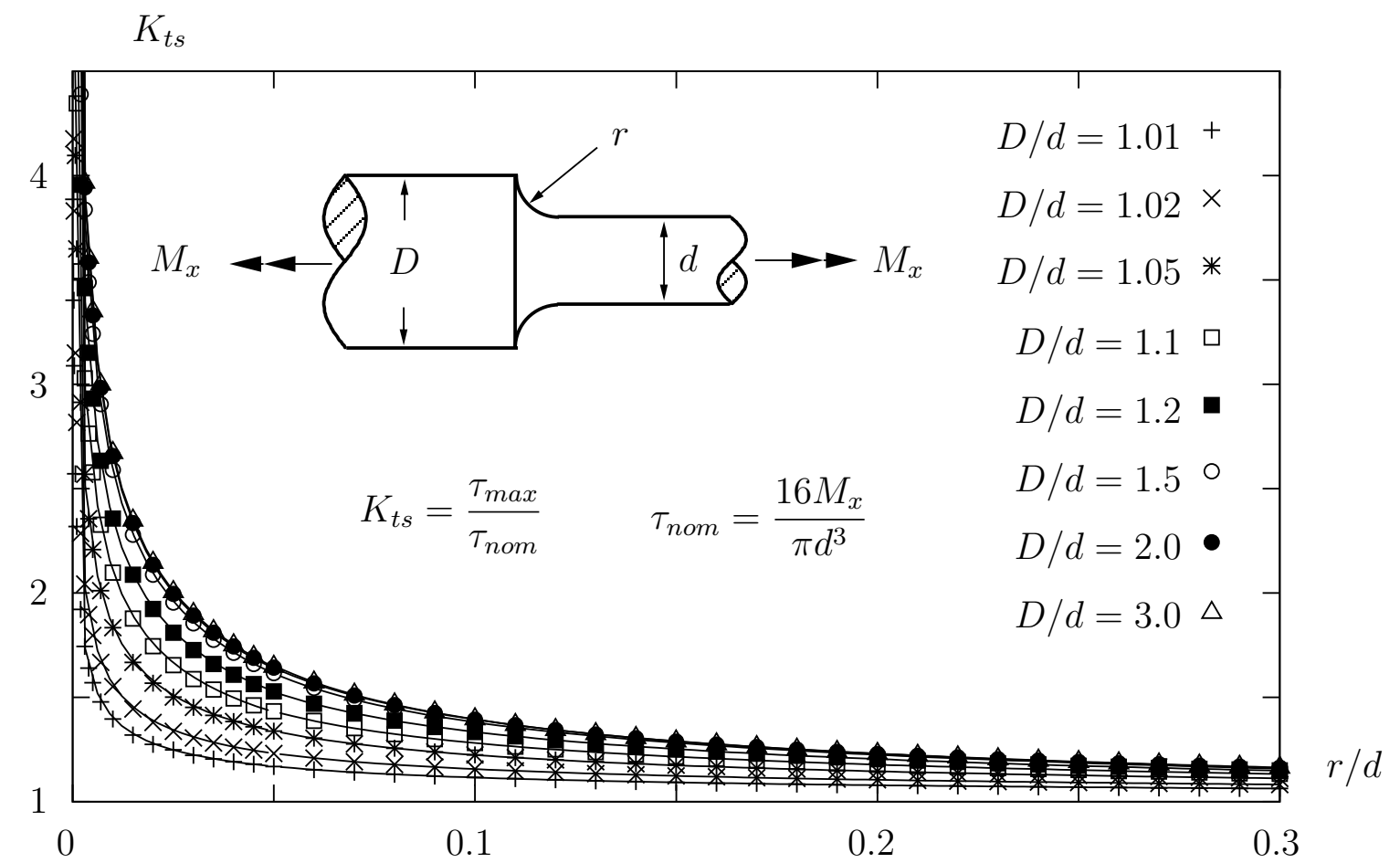

Figure 12: Chart of stress concentrations for shaft fillet in torsion, full lines are curve fits and the points are the numerical solutions.

\begin{tabular}{|c|c|c|c|c|c|c|}
\hline \multirow[b]{2}{*}{$D / d$} & \multicolumn{3}{|c|}{$r / d<0.05$} & \multicolumn{3}{|c|}{$r / d>0.05$} \\
\hline & $A$ & $B$ & $C$ & $A$ & $B$ & $C$ \\
\hline 1.010 .97359 & 0.045037 & 0.49 & 0.99941 & 0.032774 & 0.55 & \\
\hline 1.020 .93717 & 0.071826 & 0.47 & 0.99351 & 0.045898 & 0.55 & \\
\hline 1.050 .86184 & 0.12340 & 0.45 & 0.97689 & 0.069581 & 0.55 & \\
\hline 1.10 .72864 & 0.19922 & 0.42 & 0.95400 & 0.092152 & 0.55 & \\
\hline 1.20 .64628 & 0.25833 & 0.41 & 0.92983 & 0.11141 & 0.56 & \\
\hline 1.50 .46008 & 0.36970 & 0.38 & 0.90879 & 0.12412 & 0.58 & \\
\hline 2.00 .43456 & 0.38558 & 0.38 & 0.91226 & 0.12084 & 0.60 & \\
\hline 3.00 .43174 & 0.38863 & 0.38 & 0.94259 & 0.10122 & 0.65 & \\
\hline
\end{tabular}

Table 3: Values used for curve fits for torsional load.

shown that the super elliptical shape and variations of this is an excellent choice for designing a shape with minimum maximum stress.

The super ellipse (with principle axes $a$ and $b$ and super elliptical power $\eta$ ) is in parametric form given by

$$
\begin{array}{ll}
X=a \cos (t)^{(2 / \eta)}, & t \in\left[0: \frac{\pi}{2}\right] \\
Y=b \sin (t)^{(2 / \eta)}, & t \in\left[0: \frac{\pi}{2}\right]
\end{array}
$$

The optimization in the present paper is performed with the objective of having minimum maximum stress. The optimizations are performed as simple parameter studies, i.e. without sensitivity analysis. This approach is feasible here because the small number of design parameters (only three design parameters, $a, b$ and $\eta$ ). The constraints of the optimization problem are the limits to the design parameters. 


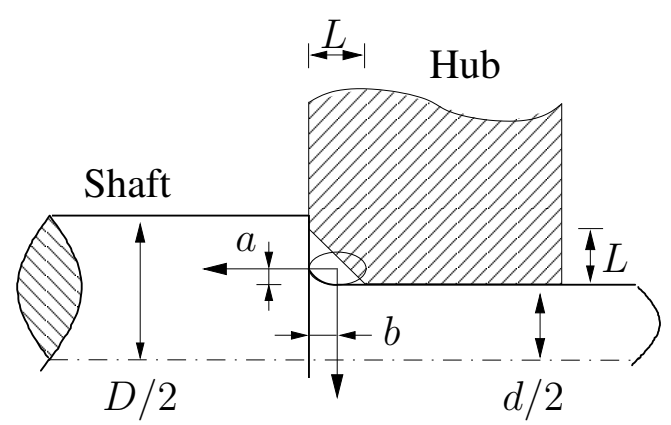

Figure 13: Part of shaft-hub connection showing the parameterization of the fillet and a hub with a $45^{\circ}$ chamfer at the outer corners with a side length $L$. Dimensions exaggerated for visualization purpose.

The reason for having a diameter change in a shaft is typically to axially constrain an attached hub. A hub typically has a $45^{\circ}$ chamfer at the outer corners with a side length $L$. The fillet in the shaft should be designed such that the hub is in axial contact with the shaft, this leads to the following constraints on the design parameters.

$$
a \leq L, \quad b \leq L, \quad \eta \geq 1
$$

The design parameterization and the constraints are illustrated in Figure 13.

To exemplify the optimization we first optimize for specific dimensions equation (8), which corresponds to a design domain side length $L=r=0.1 d$. By increasing especially the upper limit on design parameter $b$ it is possibility to minimizing the maximum stress significantly. However, relative to practical machine elements this is limited by other constraints on the overall design and therefore we choose here the more restrictive constraint value. The optimization is initially performed separately for the three different load cases. For the original circular fillet design we have the following stress concentration factors; axial load $K_{t}=2.198$, bending load $K_{t}=1.826$ and torsional load $K_{t s}=1.3903$. The optimization results are

- Axial load: $a=0.51 r, b=r, \eta=1.61$, stress reduction $14.7 \%$.

- Bending: $a=0.48 r, b=r, \eta=1.67$, stress reduction $14.2 \%$.

- Torsion: $a=0.49 r, b=r, \eta=1.73$, stress reduction $7.6 \%$.

The stress distribution along the fillet for the three design cases are combined in Figure 14 for easy comparison. It is clearly seen that overall the three designs gives a constant stress along the fillet.

For most applications the shaft loading is a combination of axial, bending and torsional load. The stress concentration factor evaluation must therefore be specified relative to an equivalent stress, e.g. von Mises stress. In practical applications, e.g. in machine elements, it is common to use the stress concentration charts for the single load case and use a conservative assumption for evaluating the combined stress concentration factor. The following assumptions are used

- The stress concentration factors for the three loads are found from the chart related to the single load cases.

- Although the maximum stress is not found in the same point for the three load cases this is assumed. 


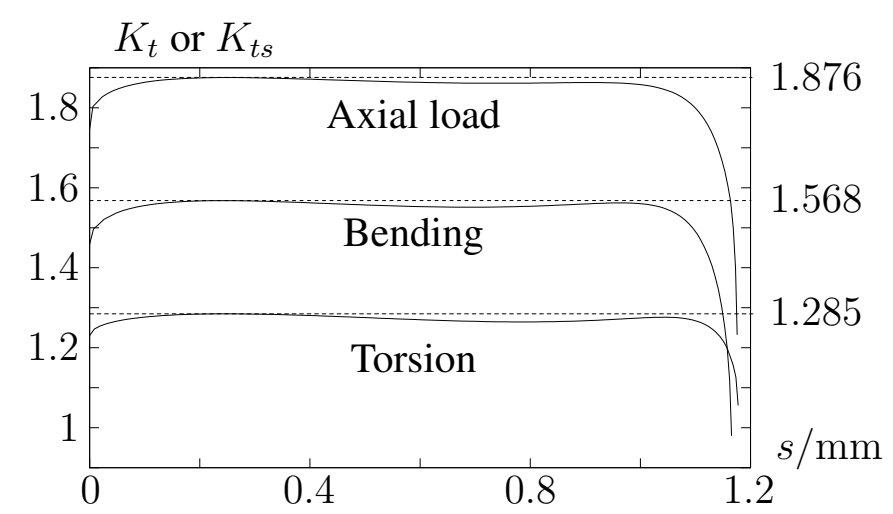

Figure 14: Stress concentration factor along fillets in shaft for three different optimized designs. Here $s$ is the arc length along the fillet.

- For bending and axial load the maximum normal stress is assumed to be in the axial direction, i.e. the $x$-axis in Figure 1.

- For torsional load the maximum shear stress is assumed to be in the plane with the $x$-axis as normal as defined in equation (3).

For a specific combination of loads (defined by the individual nominal stress) where the axial load alone is $\sigma_{\text {nom }}^{\text {axial }}=1$ and the nominal stress from bending load alone is $\sigma_{\text {nom }}^{\text {bending }}=1$ and finally that the nominal stress from torsional load alone is $\tau_{\text {nom }}^{\text {torsion }}=1$. We find the combined nominal von Mises stress

$$
\sigma_{n o m}^{v M}=\sqrt{7}
$$

Using the usual assumption for combining stress concentrations we find the stress concentration (von Mises stress)

$$
K_{v M}^{a s s u m p}=\frac{\sqrt{(2.198+1.826)^{2}+3(1.3903)^{2}}}{\sigma_{\text {nom }}^{v M}}=1.77
$$

Calculating the von Mises stress numerically for the combined load case we find the following stress concentration

$$
K_{v M}=1.64
$$

which clearly shows that the normal procedure for calculating the combined stress concentration is conservative.

Applying optimization and the elliptical shape design we find the design, $a=0.49 r, b=r$, $\eta=1.67$ giving a reduced stress concentration factor of $K_{v M}=1.45$.

The optimization result will depend on the relative amount of the three different load cases. Exemplified further with the results

- Combined load $\sigma_{\text {nom }}^{\text {axial }}=2, \sigma_{\text {nom }}^{\text {bending }}=1$ and $\tau_{\text {nom }}^{\text {torsion }}=1$ result in design $a=0.49 r$, $b=r, \eta=1.63$ and stress concentration factor of $K_{v M}=1.53$ equal to a stress reduction of $13.2 \%$. 
- Combined load $\sigma_{\text {nom }}^{\text {axial }}=1, \sigma_{\text {nom }}^{\text {bending }}=2$ and $\tau_{\text {nom }}^{\text {torsion }}=1$ result in design $a=0.48 r$, $b=r, \eta=1.64$ and stress concentration factor of $K_{v M}=1.46$ equal to a stress reduction of $12.9 \%$.

- Combined load $\sigma_{\text {nom }}^{\text {axial }}=1, \sigma_{\text {nom }}^{\text {bending }}=1$ and $\tau_{\text {nom }}^{\text {torsion }}=2$ result in design $a=0.49 r$, $b=r, \eta=1.70$ and stress concentration factor of $K_{v M}=1.36$ equal to a stress reduction of $11.0 \%$.

\subsection{Generalizing results}

The optimization in the previous section is performed for a specific size of fillet and diameter ratio. If these values change the optimal design parameter values also change. For $D / d=3$ and $L=0.1 d$ loaded by axial load, bending and torsion $\left(\sigma_{\text {nom }}^{\text {axial }}=1, \sigma_{\text {nom }}^{\text {bending }}=1\right.$ and $\left.\tau_{\text {nom }}^{\text {torsion }}=1\right)$ the optimized values are $a=0.52 r, b=r, \eta=1.62$. That corresponds to a $11.3 \%$ reduction in stress compared to the circular design.

With the found variation in the design parameter values it is difficult to make a suggestion for a new common standard for fillet design, that is applicable for the whole range of diameter variations and load variations. The results show, however, that it is possible with the super elliptical design parameterization to reduce the stress independently of the dimensions and load combinations.

If the dimensions are fixed $(d, D$ and $r$ ) but the load combination is unknown it is possible to suggest values for the design parameters that independently on the load combination will reduce the stress level compared to the circular design.

\section{Conclusion}

In the present paper stress concentration of fillets are found using a refined finite element analysis using axisymmetric harmonic elements that are capable of handling out of plane forces. This enables efficient modelling of combined loading of both axial, bending and torsional load. New charts for the standard circular fillet design are presented together with curve fits.

The influence from Poisson's ratio (often neglected) is discussed and it is shown that a difference of up to $10 \%$ in the stress level can be related to the selected Poisson's ratio. Generally the charts are presented for steel $(\nu=0.3)$.

The stress concentration factor for the combined loading is discussed and the common procedure for finding the combined stress concentration factor from the charts is shown to be conservative.

To minimize (optimize) the fillet stress the super elliptical shape is suggested. The benefits being that the design parameters are few while still being flexible enough to obtaining a design where the stress level is constant over a majority of the fillet surface. The possibility of specifying a new standard design is discussed, but due to the large variation in the overall relative dimensions and the large variation in the load combinations it is found that this is not possible. For a specific design it is shown that it is possible to reduce the stress with up to $15 \%$.

\section{Acknowledgment}

For discussions and suggestions I wish to thank Prof. Peder Klit and Prof. Pauli Pedersen. 


\section{References}

ANSYS (1970 -), Canonsburg, Pennsylvania, USA, www.ansys.com.

Bhavikatti, S. S. \& Ramakrishnan, C. V. (1979), 'Optimum shape design of shoulder fillets in tension bars and t-heads', International Journal of Mechanical Sciences 21(1), 29-39.

Dally, J. \& Riley, W. (1991), Experimental stress analysis, McGraw-Hill,.

Ding, Y. (1986), 'Shape optimization of structures: A literature survey', Computers and Structures 24(6), 985-1004.

Norton, R. L. (2000), Machine design - an integrated approach, second edn, Prentice-Hall, New Jersey 07458, USA. 1078 pages.

Parvizian, J. \& Fenner, R. (2002), 'Shape optimization of fillets by the boundary element method', Journal of Strain Analysis for Engineering Design 37(2), 93-100.

Pedersen, N. L. (2011), 'Optimization of straight-sided spline design', Archive of Applied Mechanics 81(10), 1393-1407.

Pedersen, N. L. (2013), 'Overall bolt stress optimization', Journal of Strain Analysis for Engineering Design 48(3), 155-165.

Pedersen, N. L. (2015), 'Minimizing tooth bending stress in spur gears with simplified shapes of fillet and tool shape determination', Engineering optimization 47(6), 805-824.

Pedersen, N. L. \& Pedersen, P. (2008), 'Design of notches and grooves by means of elliptical shapes', Journal of Strain Analysis for Engineering Design 43(1), 1-14.

Pedersen, P. (2000), 'On optimal shapes in materials and structures', Struct. Multidisc. Optim. 19(3), 169-182.

Pedersen, P. (2008), 'Suggested benchmarks for shape optimization for minimum stress concentration', Struct. Multidisc. Optim. 35(4), 273-283.

Pedersen, P. \& Laursen, C. L. (1982), 'Design for minimum stress concentration by finite elements and linear programming', J. Structural Mechanics 10(4), 375-391.

Pilkey, W. D. (1997), Peterson's Stress Concentration Factors, 2nd edn, John Wiley and Sons, New York, USA. 508 p.

Rolovic, R., Tipton, S. M. \& Sorem, J. R. (2001), 'Multiaxial stress concentration in filleted shafts', Journal of Mechanical Design, Transactions of the Asme 123(2), 300-303.

Sonmez, F. O. (2009), 'Optimal shape design of shoulder fillets for flat and round bars under various loadings', Proceedings of the Institution of Mechanical Engineers Part C-journal of Mechanical Engineering Science 223(8), 1741-1754.

Tipton, S., Sorem, J. \& Rolovic, R. (1996), 'Updated stress concentration factors for filleted shafts in bending and tension', Journal of Mechanical Design 118(3), 321-327.

Van Miegroet, L. \& Duysinx, P. (2007), 'Stress concentration minimization of 2d filets using x-fem and level set description', Structural and Multidisciplinary Optimization 33(45), 425-438. 